\title{
GESTÃO DA INFORMAÇÃO EM TEMPOS DE CRISE: A EXPERIÊNCIA DA UNIVERSIDADE FEDERAL DO TOCANTINS NA PANDEMIA COVID-19
}

Information management in times of crisis: the experience of the Federal University of Tocantins in pandemia covid-19

Gestión de información en tiempos de crisis: la experiencia de la Universidad Federal de Tocantinas en pandemia covid-19

\section{Kleber Abreu Sousa ${ }^{*}$, Márcia Thiely de Macedo ${ }^{2}$, André Massa $^{3}$, Débora de Oliveira Sousa ${ }^{4}$ Clarete De Itoz ${ }^{5}$}

${ }^{1}$ Programa de Pós Graduação em Propriedade Intelectual e Transferência de Tecnologia para a Inovação, Universidade Federal do Tocantins, Câmpus de Palmas, Tocantins, Brasil.

${ }^{2}$ Programa de Pós Graduação em Propriedade Intelectual e Transferência de Tecnologia para a Inovação, Universidade Federal do Tocantins, Câmpus de Palmas, Tocantins, Brasil.

${ }^{3}$ Programa de Pós Graduação em Comunicação, Faculdade Cásper Líbero, São Paulo, Brasil.

${ }^{4}$ Programa de Pós Graduação em Administração de Empresas - Doutorado, Centro Universitário FEI, Câmpus de São Paulo, São Paulo, Brasil.

${ }^{5}$ Programa de Pós Graduação em Contabilidade - Mestrado, Faculdade Visconde de Cairu, Salvador, Brasil.

*Correspondência: Superintendência de Comunicação, Bloco III, sala 14. Av. NS 15, 109 Norte, Palmas, Tocantins, Brasil.CEP:77.010-090.e-mail kleberabreu@uft.edu.br.

\section{RESUMO}

Qualquer situação, diferente do comum, que afete ou ameace afetar negativamente as Instituições, seja sua função, sua finalidade, suas atividades, sua estabilidade e sua imagem, deve ser tratada como crise ou crise em potencial. As ações de comunicação em situações de crise além de preservar a imagem e a reputação da Instituição, não devem ignorar ou menosprezar, nestas circunstâncias, sua finalidade principal de atender ao interesse público. No ambiente universitário, local reconhecido como ambiente de forte contestação, reflexão e análise da realidade, faz-se ainda mais necessária a utilização das plataformas de conteúdo disponíveis, com o propósito de esclarecer e comunicar à comunidade as informações relevantes em tempos de crise. Dentro desse contexto, questiona-se: qual o efetivo alcance das informações que são produzidas nas plataformas de conteúdo das Universidades sobre a comunidade, em tempos de crise? Esta pesquisa revela a experiência da Universidade Federal do Tocantins - UFT no período inicial da pandemia covid -19, em relação à gestão das informações. À luz da pesquisa exploratória, o estudo concluiu que a gestão integrada das plataformas de conteúdo institucionais precisa estar conectada e a mensuração do volume de publicações, bem como o alcance destas, precisa ser continuamente monitorado, sobretudo, em tempos de crise.

Palavras-chave: pandemia, covid-19, informação

\section{ABSTRACT}

Any situation, other than the ordinary, that affects or threatens to negatively affect the Institutions, be it their function, purpose, activities, stability and image, must be treated as a crisis or potential crisis. Communication actions in crisis situations, in addition to preserving the Institution's image and reputation, must not ignore or underestimate, in these circumstances, its main purpose of serving the public interest. In the university environment, a place recognized as an environment of strong contestation, reflection and analysis of reality, it becomes even more necessary to use the available content platforms, in order to clarify and communicate to the community the relevant 
information in times of crisis. Within this context, the question is: what is the effective reach of the information that is produced on the Universities' content platforms about the community, in times of crisis? This research reveals the experience of the Federal University of Tocantins - UFT in the pandemic covid -19, regarding information management. In the light of exploratory research, the study concluded that the integrated management of institutional content platforms needs to be well articulated and the measurement of the volume of publications, as well as the reach of these, needs to be continuously monitored, especially in times of crisis.

Keywords: pandemic, covid-19, information

\section{RESUMEN}

Cualquier situación, distinta de la ordinaria, que afecte o amenace con afectar negativamente a las Instituciones, ya sea su función, propósito, actividades, estabilidad e imagen, debe tratarse como una crisis o crisis potencial. Las acciones de comunicación en situaciones de crisis, además de preservar la imagen y la reputación de la institución, no deben ignorar ni subestimar, en estas circunstancias, su principal objetivo de servir al interés público. En el entorno universitario, un lugar reconocido como un entorno de fuerte desafío, reflexión y análisis de la realidad, se hace aún más necesario utilizar las plataformas de contenido disponibles, con el fin de aclarar y comunicar la información relevante en tiempos de crisis a la comunidad. En este contexto, la pregunta es: ¿cuál es el alcance efectivo de la información que se produce en las plataformas de contenido de las universidades sobre la comunidad, en tiempos de crisis? Esta investigación revela la experiencia de la Universidad Federal de Tocantins - UFT en la pandemia covid -19, con respecto al manejo de la información. A la luz de la investigación exploratoria, el estudio concluyó que la gestión integrada de las plataformas de contenido institucional debe estar bien articulada y la medición del volumen de publicaciones, así como el alcance de estas, debe monitorearse continuamente, especialmente en tiempos de crisis.

Descriptores: pandemia, covid-19, información

\section{INTRODUÇÃO}

Situações de emergência em saúde pública, demandam o que é chamado de "comunicação de risco", cujo objetivo é informar e conscientizar o público, sobretudo quando há alta incidência de alguma doença ou epidemia. A gestão e o controle dessas situações adversas envolvem, invariavelmente, ações e estratégias de comunicação institucional. No ambiente Universitário essa realidade se torna ainda mais evidente, tanto pela natureza da Instituição, quanto pela expectativa que a sociedade coloca nela. De acordo com Barichello (2004, p. 8) "o destino da Universidade está ligado às relações comunicacionais que a comunidade universitária conseguir estabelecer com a sociedade do seu tempo". Segundo a autora, numa época em que os meios de comunicação reorganizam a sociedade, supõe-se que a universidade precisaria utilizar-se de dispositivos para torná-la visível e legítima perante o seu tempo Corroborando com essa afirmativa, Kunsch (1986, p.40) acredita que “(...) não se pode mais pensar numa comunicação interna fechada e restrita ao universo institucional, mas composta de um conteúdo que leva em conta as interferências do ambiente externo." Em tempos de crise, precisa-se ainda reafirmar que a comunicação é um processo dialógico, sistêmico e orgânico. Dessa forma, por mais que haja um direcionamento institucional, entende-se que a qualidade e efetividade da comunicação dependem de todos os envolvidos e que os canais de comunicação precisam ser os mais capilarizados possíveis e com linguagens que tenham conexões com o público a que se destinam. E é nesse sentido que esse artigo busca apresentar a quantidade $\mathrm{e}$ alcance das publicações institucionais da UFT, em tempos de pandemia Covid-19.

Revista Desafios - v7, n. Supl. COVID-19, 2020 


\section{MATERIAIS E MÉTODOS}

Enquanto método de pesquisa foi escolhida a pesquisa exploratória por ser a mais adequada ao objeto em questão, pelo fato de possuir ainda modesto conhecimento acumulado ou estudos realizados. (GIL, 2008). Segundo (LAKATOS e MARCONI, 2003, p. 155), pesquisa é o caminho percorrido no estudo de um objeto, para conhecer a realidade ou verdades parciais, com tratamento científico específico. Neste caso, a gestão das informações na UFT em tempos de Covid19. Também foram utilizados, de maneira subsidiária, a pesquisa bibliográfica e a pesquisa documental. A primeira no tocante à literatura sobre gestão da informação em tempos de crise. A segunda pela análise dos documentos, indicadores e índices de acesso a conteúdos informacionais internos. Em relação aos métodos de procedimentos aplicados no trabalho, foi adotado o método de estudo de caso, haja vista o objetivo de analisar aspectos envolvidos na gestão da informação no caso específico da Universidade Federal do Tocantins - UFT, à luz da pandemia Covid-19. O tratamento dos dados levantados na pesquisa foram realizados utilizando as ferramentas estatísticas dos softwares internos da UFT, bem como o ferramental das mídias sociais avaliadas, para a emissão das tabelas.

\section{RESULTADOS E DISCUSSÃO}

\section{O uso das plataformas de conteúdo da Universidade}

Federal do Tocantins - UFT em tempos de crise

A fim de se evitar informações divergentes, confusas e contraditórias, recomenda-se, em situações de crise, a centralização de fluxos e canais de divulgação de informações. Além de preservar a imagem e a reputação da Instituição, as ações de comunicação em situações de crise não devem ignorar ou menosprezar, nestas circunstâncias, sua finalidade principal de atender ao interesse público. Assim, recomenda-se que sejam empenhados esforços de comunicação, em situações de crise, para a prestação de serviços e a divulgação de informações de utilidade pública. Conforme já comentando, a gestão das informações em tempos de crise requer especial cuidado por parte das Universidades, uma vez que estes ambientes são reconhecidos como a "casa da ciência"; sendo assim, a expectativa por informações precisas advindas das Universidades, é alta. É nesse sentido que os resultados desse estudo apresentam a dinâmica das informações institucionais no início da pandemia Covid-19, por meio de duas plataformas de conteúdo da Universidade Federal do Tocantins -UFT.

Além da qualidade da informação, outro ponto que pouco é abordado pela literatura relacionada à comunicação Institucional, porém é imprescindível em tempos de crise, é o "alcance daquilo que se comunica". Publicar conteúdos relevantes e com qualidade técnica não é suficiente em cenários de crise. A informação precisa chegar à comunidade acadêmica pelos mais capilarizados meios possíveis, garantindo assim, o acesso da informação ao maior número de pessoas. Com o propósito de dar maior capilaridade à comunicação no período da pandemia Covid - 19, a UFT utilizou todas as suas plataformas de conteúdo, com destaque para o Portal UFT e mídias digitais. A seguir será apresentado o levantamento do quantitativo de conteúdos publicados no período da pandemia Covid - 19, bem como o alcance de cada um, de acordo com as 2 plataformas mais utilizadas:

Plataforma I) Portal UFT - O portal institucional é um espaço virtual multimídia que agrega as principais informações institucionais, bem como notícias, tópicos 
e links relacionados às atividades da Instituição. É o ponto de convergência dos conteúdos institucionais da UFT em seus diferentes tipos e formatos de mídia, sendo o canal oficial para a divulgação primária de notícias, comunicados, documentos e informações oficiais relacionadas às atividades acadêmicas e administrativas da Instituição. O gráfico expresso na figura 1 demonstra o alcance ou seja, o total de acessos que essa plataforma apresentou no exercício de 2019:

Figura 1) Total de acessos ao portal (2019)

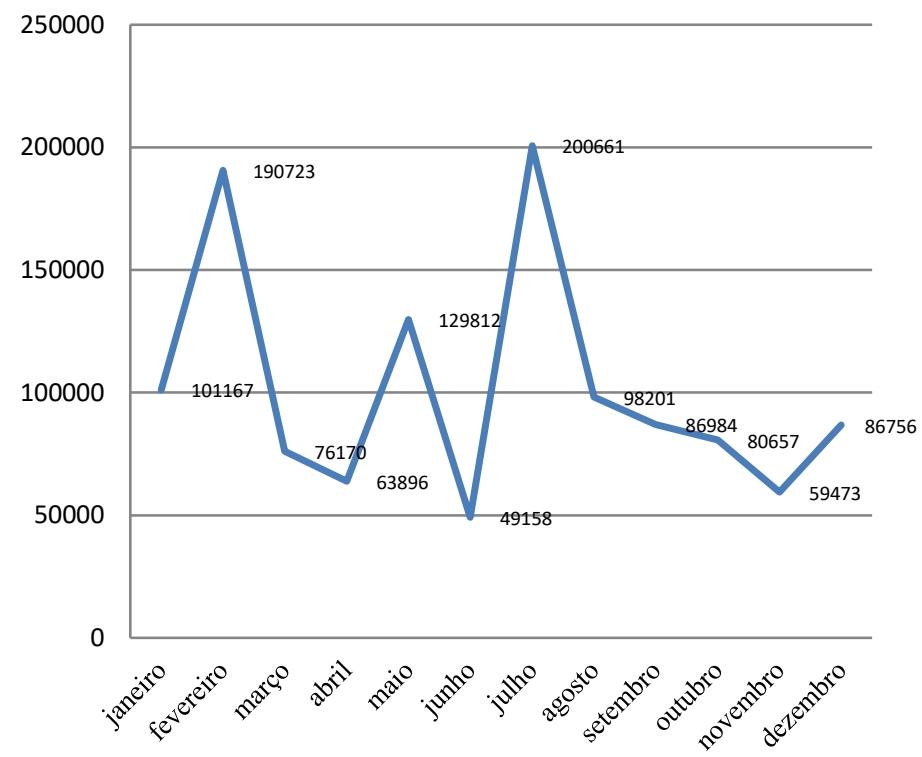

Fonte: Relatório de indicadores - SUCOM

Com base na figura 1 , o portal teve um total de crescimento de $0,2957 \%$ no total de acessos semestral na comparação 2019/1 com 2019/2 (610.926 no $1^{\text {o }}$ semestre e 612.732 no $2^{\circ}$ semestre), somando um total de acessos no ano de 1.223.658. Considera-se esse número um quantitativo representativo tendo em vista o fato de que a comunidade acadêmica da UFT é composta de aproximadamente 20 mil pessoas.

Esta plataforma, portanto, teve fundamental importância na divulgação das informações relativas à pandemia Covid - 19, no ambiente universitário. Sem contar que no portal da UFT há um ícone de chamada direta para as ações covid, o que facilita a comunicação direta sobre o assunto e demais notícias correlatas. Partindo da premissa de que o alcance daquilo que se comunica é tão importante quanto à qualidade técnica do que é noticiado, na tabela 1 é apresentado o número de notícias relacionadas à pandemia Covid -19 publicadas nesta plataforma de conteúdo, bem como o seu alcance:

Tabela 1. Relação e alcance das publicações de matérias (período de 15 março a 05 de abril)

\begin{tabular}{|c|c|c|}
\hline Matéria & Data & Acessos \\
\hline $\begin{array}{l}\text { Atividades da Universidade da } \\
\text { Maturidade (UMA) estão suspensas até } \\
23 \text { de março }\end{array}$ & 16.03 .20 & 511 \\
\hline $\begin{array}{l}\text { Seleção para aluno especial do PPGDire } \\
\text { é cancelada devido à crise do } \\
\text { coronavírus }\end{array}$ & 16.03 .20 & 373 \\
\hline $\begin{array}{l}\text { UFT divulga orientações e suspende } \\
\text { atividades; Comitê fará avaliação diária } \\
\text { da situação }\end{array}$ & 16.03 .20 & 13018 \\
\hline $\begin{array}{l}\text { Prograd altera } \\
\text { matrícula dos } \\
\text { vigentes }\end{array}$ & 17.03 .20 & 1337 \\
\hline $\begin{array}{l}\text { O que você precisa saber sobre a Covid- } \\
19, \text { o novo Coronavírus }\end{array}$ & 17.03 .20 & 782 \\
\hline $\begin{array}{l}\text { Coordenação divulga nota sobre a } \\
\text { suspensão das aulas }\end{array}$ & 17.03 .20 & 941 \\
\hline $\begin{array}{l}\text { Programa Radar terá entrevista com } \\
\text { infectologista, nesta quarta (18) }\end{array}$ & 17.03 .20 & 232 \\
\hline $\begin{array}{l}\text { Proex informa que entrevistas para } \\
\text { verificação de cotas estão suspensas }\end{array}$ & 17.03 .20 & 289 \\
\hline $\begin{array}{l}\text { Reitor reforça necessidade de } \\
\text { isolamento social para conter avanço do } \\
\text { Coronavírus }\end{array}$ & 18.03 .20 & 1377 \\
\hline $\begin{array}{l}\text { Instrução } \quad \text { Normativa } \\
\text { orientações sobre suspensão das aulas } \\
\text { presenciais }\end{array}$ & 18.03 .20 & 1595 \\
\hline $\begin{array}{l}\text { UFT suspende aplicação de provas do } \\
\text { Vestibular } 2020 / 2 \text { e Seleção da EaD } \\
2020 / 2\end{array}$ & 18.03 .20 & 4012 \\
\hline $\begin{array}{l}\text { Cineclube suspende exibição de filmes e } \\
\text { debates por tempo indeterminado }\end{array}$ & 18.03 .20 & 141 \\
\hline $\begin{array}{l}\text { RU de Palmas interrompe serviços; } \\
\text { Proest dará novas orientações na } \\
\text { segunda }(23)\end{array}$ & 20.03 .20 & 312 \\
\hline $\begin{array}{l}\text { UMA cria projeto para auxiliar os } \\
\text { idosos nesta fase de isolamento social }\end{array}$ & 23.03 .20 & 340 \\
\hline $\begin{array}{l}\text { Câmpus de Gurupi produzirá álcool em } \\
\text { gel para distribuir a entidades e à } \\
\text { população }\end{array}$ & 23.03 .20 & 563 \\
\hline $\begin{array}{l}\text { Visitas a pacientes e consultas } \\
\text { ambulatoriais estão temporariamente } \\
\text { suspensas no HDT-UFT }\end{array}$ & 23.03 .20 & 138 \\
\hline $\begin{array}{l}\text { UFT atualiza orientações; Proest e } \\
\text { Progedep divulgam novas diretrizes }\end{array}$ & 23.03 .20 & 2577 \\
\hline $\begin{array}{l}\text { Proest divulga novas diretrizes sobre } \\
\text { RUs e Auxílios Emergenciais }\end{array}$ & 23.03 .20 & 4491 \\
\hline
\end{tabular}




\begin{tabular}{|c|c|c|}
\hline $\begin{array}{l}\text { Lives em rede social abordarão sobre } \\
\text { contaminação da Covid-19 através de } \\
\text { alimentos }\end{array}$ & 24.03 .20 & 296 \\
\hline $\begin{array}{l}\text { Projeto Ecoa/SUS divulga resultado de } \\
\text { seleção e suspende segunda etapa }\end{array}$ & 25.03 .20 & 136 \\
\hline $\begin{array}{l}\text { UFT disponibiliza acesso à Biblioteca } \\
\text { Virtual Pearson para comunidade } \\
\text { universitária }\end{array}$ & 26.03 .20 & 2392 \\
\hline $\begin{array}{l}\text { Assista à transmissão ao vivo da } \\
\text { Reunião do Conselho } \\
\text { (Consuni) }\end{array}$ & 30.03 .20 & 1037 \\
\hline $\begin{array}{l}\text { Conselho Universitário delibera pela } \\
\text { suspensão do Calendário Acadêmico } \\
2020\end{array}$ & 30.03 .20 & 6453 \\
\hline $\begin{array}{l}\text { Confira a lista de estudantes aprovados } \\
\text { para o } \quad \text { Auxílio } \quad \text { Alimentação } \\
\text { Emergencial }\end{array}$ & 31.03 .20 & 881 \\
\hline $\begin{array}{l}\text { UFT confecciona protetores faciais para } \\
\text { profissionais da saúde pública de } \\
\text { Palmas }\end{array}$ & 31.03 .20 & 1293 \\
\hline $\begin{array}{l}\text { PPGHispam prorroga inscrições para } \\
\text { seleção ao mestrado até } 30 \text { de abril }\end{array}$ & 31.03 .20 & 361 \\
\hline $\begin{array}{l}\text { Funcionários terceirizados da UFT } \\
\text { recebem orientações preventivas da } \\
\text { Covid-19 }\end{array}$ & 01.04 .20 & 115 \\
\hline $\begin{array}{l}\text { Ebserh lança edital para contratação } \\
\text { temporária de } 6 \text { mil profissionais }\end{array}$ & 03.04 .20 & 313 \\
\hline $\begin{array}{l}\text { Produção de álcool em gel para } \\
\text { combate à Covid-19 é ampliada na } \\
\text { UFT }\end{array}$ & 06.04 .20 & 46 \\
\hline Total & & 46.352 \\
\hline
\end{tabular}

Fonte: Relatório de indicadores - SUCOM

De acordo com o que apresenta a tabela 1, foram produzidas 29 matérias relacionadas ao coronavírus.

Vale ressaltar que as matérias relacionadas à pandemia e suas consequências, envolvem diversos eixos da instituição, como atividades administrativas, atividades de ensino, mobilidade, etc... No período de 20 de março a 05 de abril, foi constatado nas 29 matérias, 46.352 acessos; tem-se portanto uma média de aproximadamente 1.600 acessos por matéria. O índice de acesso é considerado bom se comparado ao número de indivíduos que fazem parte da comunidade acadêmica.

Plataforma II) Mídias Sociais - A UFT ainda está presente com perfis, páginas ou canais oficiais no Facebook, no Twitter, no Instagram, no YouTube e no Linkedin. Em todas as redes, a Universidade identificase como "UFT Oficial". Os conteúdos publicados são direcionados conforme o público, os recursos e funcionalidades existentes em cada rede social, sendo possíveis interações como "seguir", "curtir", "compartilhar" e "comentar" conteúdos, bem como o envio direto de mensagens à Instituição. No caso particular do Facebook e Instagram, mídias de maior alcance da UFT, em tempos de pandemia Covid -19, um grande volume de informações foi publicado com o propósito de atingir cada vez mais, um número maior de estudantes, público alvo mais presente nesse tipo de mídia. A tabela 2 apresenta o alcance obtido a partir dessas publicações:

Tabela 2. Total de postagens e reações nas redes instagram e facebook (período de 20 março a 05 de abril)

\begin{tabular}{|c|c|c|c|}
\hline Postagem & Curtidas & $\begin{array}{l}\text { Compart. } \\
\text { Facebook }\end{array}$ & $\begin{array}{c}\text { Alcance e } \\
\text { envolvimento } \\
\text { Facebook }\end{array}$ \\
\hline $\begin{array}{ll}\text { Vídeo } & 1) \\
\text { Campanha } & \text { de } \\
\text { prevenção } & \text { ao } \\
\text { Covid - 19 } & \end{array}$ & $\begin{array}{c}\text { Facebook } \\
07 \\
\\
\text { Instagram } \\
865 \\
\text { visualizações } \\
\text { e } 64 \text { curtidas }\end{array}$ & 01 & $\begin{array}{c}783 \text { pessoas } \\
\text { alcançadas } \\
30 \\
\text { envolvimentos }\end{array}$ \\
\hline $\begin{array}{l}\text { Vídeo } \\
\text { Produção de } \\
\text { protetores } \\
\text { faciais na UFT }\end{array}$ & $\begin{array}{c}\text { Facebook } \\
104 \\
\text { Instagram } \\
6.523 \\
\text { visualizações } \\
\text { e } 1.112 \\
\text { curtidas } \\
\end{array}$ & 100 & $\begin{array}{c}9.132 \text { pessoas } \\
\text { alcançadas } \\
1.084 \\
\text { envolvimentos }\end{array}$ \\
\hline $\begin{array}{lr}\text { Vídeo } & \text { 3) } \\
\text { Mensagem } & \\
\text { sobre } & \\
\text { suspensão } & \text { das } \\
\text { aulas } & \text { em } \\
\text { função } & \text { do } \\
\text { covid -19 } & \end{array}$ & $\begin{array}{c}\text { Facebook } \\
32 \\
\text { Instagram } \\
4.294 \\
\text { visualizações } \\
\text { e } 561 \\
\text { curtidas }\end{array}$ & 09 & $\begin{array}{c}2.379 \text { pessoas } \\
\text { alcançadas } \\
246 \\
\text { envolvimentos }\end{array}$ \\
\hline $\begin{array}{lr}\text { Foto 1) Galeria } \\
\text { com } 4 \text { fotos } \\
\text { sobre } \\
\text { produção de } \\
\text { álcool em gel } \\
\text { em Gurupi }\end{array}$ & $\begin{array}{c}\text { Facebook } \\
246 \\
\text { Instagram } \\
891\end{array}$ & 68 & $\begin{array}{c}9.148 \text { pessoas } \\
\text { alcançadas } \\
1.173 \\
\text { envolvimentos }\end{array}$ \\
\hline $\begin{array}{l}\text { Vídeo 4) } \\
\text { Higienização } \\
\text { correta das }\end{array}$ & $\begin{array}{l}\text { Facebook } \\
22 \\
\text { Instagram }\end{array}$ & 07 & $\begin{array}{c}1.875 \text { pessoas } \\
\text { alcançadas } \\
102 \\
\text { envolvimentos }\end{array}$ \\
\hline
\end{tabular}




\begin{tabular}{|c|c|c|c|}
\hline $\begin{array}{lr}\text { mãos } & \text { em } \\
\text { tempos } & \text { de } \\
\text { Covid }-19 & \end{array}$ & $\begin{array}{c}1.225 \\
\text { visualizações } \\
\text { e } 95 \text { curtidas }\end{array}$ & & \\
\hline $\begin{array}{ll}\text { Card } & 1) \\
\text { Medidas } & \text { de } \\
\text { proteção } & \text { ao } \\
\text { Covid-19 } & \end{array}$ & $\begin{array}{l}\text { Facebook } \\
\quad 32 \\
\text { Instagram } \\
529\end{array}$ & 12 & $\begin{array}{c}2.566 \text { pessoas } \\
\text { alcançadas } \\
251 \\
\text { envolvimentos }\end{array}$ \\
\hline $\begin{array}{lr}\text { Foto 2) } & \text { Galeria } \\
\text { com } & \text { fotos } \\
\text { sobre } & \text { a } \\
\text { produção de } & \text { décool em } \\
\text { ael } \\
\text { em Gurupi }\end{array}$ & $\begin{array}{c}\text { Facebook } \\
177 \\
\text { Instagram } \\
1.519\end{array}$ & 59 & $\begin{array}{c}7.227 \text { pessoas } \\
\text { alcançadas } \\
666 \\
\text { envolvimentos }\end{array}$ \\
\hline $\begin{array}{l}\text { Card } \\
\text { Medidas } \\
\text { administrativas } \\
\text { relativas ao } \\
\text { Covid - } 19\end{array}$ & $\begin{array}{l}\text { Facebook } \\
07 \\
\\
\text { Instagram } \\
250\end{array}$ & 00 & $\begin{array}{c}744 \text { pessoas } \\
\text { alcançadas } \\
45 \\
\text { envolvimentos }\end{array}$ \\
\hline $\begin{array}{l}\text { Card 3) } \\
\text { Auxílios } \\
\text { Emergenciais }\end{array}$ & $\begin{array}{c}\text { Facebook } \\
19 \\
\text { Instagram } \\
281\end{array}$ & 10 & $\begin{array}{c}1.993 \text { pessoas } \\
\text { alcançadas } \\
157 \\
\text { envolvimentos }\end{array}$ \\
\hline $\begin{array}{lr}\text { Card } & 4 \text { ) } \\
\text { Suspensão } & \text { do } \\
\text { Vestibular } & \text { em } \\
\text { função } & \text { do } \\
\text { Covid - 19 } & \end{array}$ & $\begin{array}{l}\text { Facebook } \\
\quad 47 \\
\text { Instagram } \\
1.264\end{array}$ & 06 & $\begin{array}{c}2.556 \text { pessoas } \\
\text { alcançadas } \\
227 \\
\text { envolvimentos }\end{array}$ \\
\hline $\begin{array}{l}\text { Card 5) } \\
\text { Interrupção } \\
\text { dos serviços do } \\
\text { RU }\end{array}$ & $\begin{array}{c}\text { Facebook } \\
12 \\
\\
\text { Instagram } \\
338\end{array}$ & 01 & $\begin{array}{c}974 \text { pessoas } \\
\text { alcançadas } \\
46 \\
\text { envolvimentos }\end{array}$ \\
\hline $\begin{array}{lr}\text { Card } & 6) \\
\text { Suspensão } & \text { dos } \\
\text { processos } & \\
\text { seletivos } & \text { em } \\
\text { função } & \text { da } \\
\text { pandemia } & \\
\end{array}$ & $\begin{array}{c}\text { Facebook } \\
30 \\
\\
\text { Instagram } \\
1.343\end{array}$ & 18 & $\begin{array}{c}2.753 \text { pessoas } \\
\text { alcançadas } \\
216 \\
\text { envolvimentos }\end{array}$ \\
\hline $\begin{array}{lr}\text { Card } & 7 \text { ) } \\
\text { Suspensão } & - \\
\text { Entrevista } & \text { de } \\
\text { verificação } & \text { de } \\
\text { quotas } & \end{array}$ & $\begin{array}{l}\text { Facebook } \\
15 \\
\text { Instagram } \\
251\end{array}$ & 06 & $\begin{array}{c}1.617 \text { pessoas } \\
\text { alcançadas } \\
56 \\
\text { envolvimentos }\end{array}$ \\
\hline $\begin{array}{l}\text { Vídeo 5) } \\
\text { Interrupção } \\
\text { dos serviços do } \\
\text { RU }\end{array}$ & $\begin{array}{c}\text { Facebook } \\
112 \\
\\
\text { Instagram } \\
6.431 \\
\text { visualizações } \\
\text { e } 955 \\
\text { curtidas } \\
\end{array}$ & 69 & $\begin{array}{c}7.438 \text { pessoas } \\
\text { alcançadas } \\
703 \\
\text { envolvimentos }\end{array}$ \\
\hline $\begin{array}{ll}\text { Card } & 8 \text { ) } \\
\text { Suspensão } & \text { de } \\
\text { matrículas } & \end{array}$ & $\begin{array}{c}\text { Facebook } \\
13 \\
\text { Instagram } \\
199\end{array}$ & 06 & $\begin{array}{c}1.702 \text { pessoas } \\
\text { alcançadas } \\
95 \\
\text { envolvimentos }\end{array}$ \\
\hline
\end{tabular}

\begin{tabular}{|c|c|c|c|}
\hline $\begin{array}{l}\text { Card 9) Radar } \\
\text { da Ciência - } \\
\text { Coronavírus }\end{array}$ & $\begin{array}{c}\text { Facebook } \\
14 \\
\text { Instagram } \\
275\end{array}$ & 06 & $\begin{array}{c}1.051 \text { pessoas } \\
\text { alcançadas } \\
42 \\
\text { envolvimentos }\end{array}$ \\
\hline $\begin{array}{l}\text { Card 10) } \\
\text { Campanha } \\
\text { Coronavírus }\end{array}$ & $\begin{array}{c}\text { Facebook } \\
\quad 36 \\
\\
\text { Instagram } \\
747\end{array}$ & 25 & $\begin{array}{c}2.875 \text { pessoas } \\
\text { alcançadas } \\
387 \\
\text { envolvimentos }\end{array}$ \\
\hline
\end{tabular}

Fonte: Relatório de indicadores - SUCOM

Conforme dispõe a tabela 2, sobre o total de postagens e reações nas redes instagram e facebook com conteúdos relacionados ao coronavírus, no período de 20 março a 05 de abril, observou-se um maior engajamento nos vídeos postados e também nos conteúdos expressos em cards, que tinham um design mais lúdico. Esse direcionamento se deu a partir do levantamento do perfil do público alvo da UFT :

Figura 2) Perfil do público - alvo (instagram)

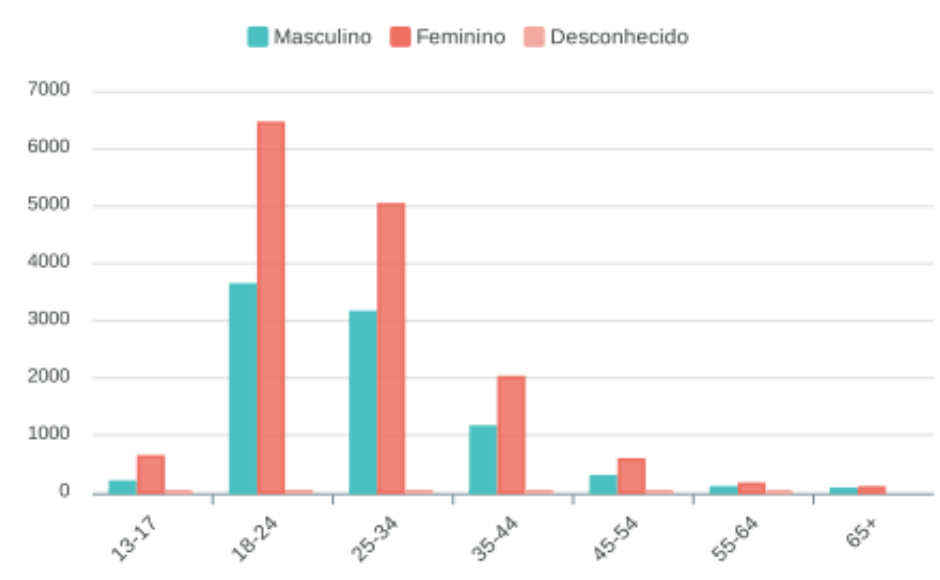

Fonte: Relatório de indicadores - SUCOM

Figura 3) Perfil do público - alvo (facebook)

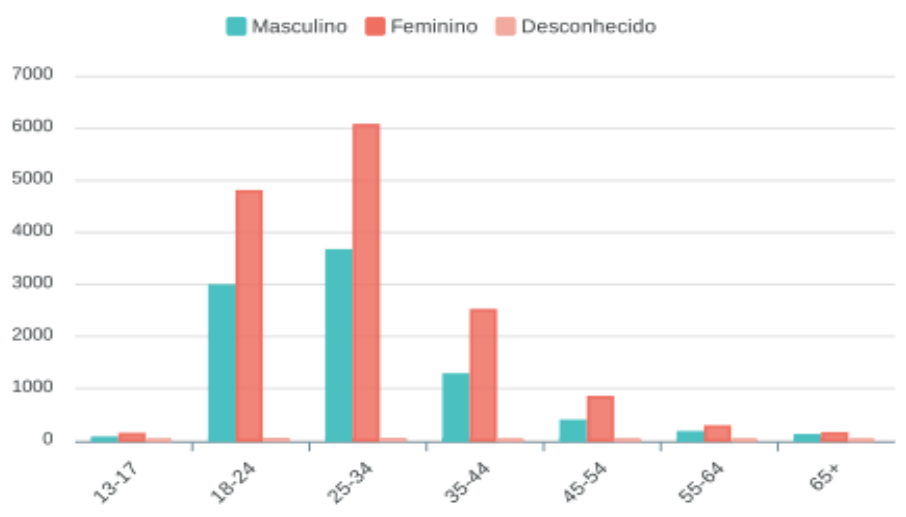


Fonte: Relatório de indicadores - SUCOM

De acordo com as figuras 2 e 3 , o público alvo da UFT nas plataformas instagram e facebook é relativamente parecido. A principal diferença é que no instagram o público majoritário possui idade entre 18 a 24 anos, e a grande maioria dos seguidores é do sexo feminino. De forma bem parecida, na plataforma facebook o público majoritário possui de 25 a 34 anos, mais velho, portanto, do que o público do instagram, e também apresenta um maior número de seguidores do sexo feminino.

\section{CONCLUSÃO}

As respostas à sociedade em situações de crise devem ser cautelosas, ágeis, claras e precisas, porém, tão importante quanto a clareza das informações, é o alcance delas, as suas penetrações. Por isso, nunca foi tão necessária a gestão da comunicação, em tempos de crise, sobretudo, em se tratando de um problema de saúde pública, como é o caso do Covid-19,

Realizar o levantamento de indicadores, como foi o caso desse trabalho, sobre o número de matérias publicadas e postagens realizadas nas plataformas de conteúdo da Instituição, bem como o alcance de cada publicação, constituem fatores fundamentais para o redirecionamento das ações integradas de comunicação institucional.

Todos os autores declararam não haver qualquer potencial conflito de interesses referente a este artigo.

\section{REFERÊNCIAS}

BARICHELLO, Eugenia Mariano da Rocha. Relações comunicacionais entre a universidade e a sociedade.
Para a gestão da comunicação, o entendimento de que o público mais representativo das redes sociais institucionais é o público jovem, do sexo feminino, traz consigo um arcabouço de informações que permitem adequar a linguagem de uma forma mais própria ao atendimento do público - alvo. Acredita-se que o expressivo número de acessos nos conteúdos publicados nas redes sociais, se deu em grande medida pelo fato da linguagem do material publicado estar mais conectada com o público específico.

Revista Iberoamericana de Educação, Madrid, n. 33-35, 2004. Disponível em: <http://www.rieoei.org/deloslectores/717Rocha.PDF >. Acesso em: 10 mar. 2020.

GIL, A. C. Como elaborar projetos de pesquisa. $4^{\mathrm{a}}$. ed. São Paulo: Atlas, 2002.

KUNSCH, Margarida Maria. Planejamento de Relações Públicas na Comunicação Integrada. São Paulo: Ed Summus, 1986.

LAKATOS, E. M.; MARCONI, M. D. A. Fundamentos de metodologia científica. São Paulo: Atlas, 2003.

NASSAR, Paulo; FIGUEIREDO, Rubens. O que é comunicação empresarial. São Paulo: Ed. Brasiliense, 2006. REZENDE, Eduardo. Gerenciamento de marcas: como planejar e criar marcas competitivas. 2008. Trabalho de Conclusão de Curso (Graduação em Comunicação Social) - Faculdade de Comunicação Social, Universidade Federal de Juiz de Fora, Juiz de Fora, 2008.

TORQUATO, Francisco Gaudêncio. Comunicação empresarial/comunicação institucional: conceitos, estratégias, sistemas, estrutura, planejamento e técnincas. São Paulo: Ed. Summus, 1986. 